\title{
Review of: "Dominance of the residential sector in Chinese black carbon emissions as identified from downwind atmospheric observations during the COVID-19 pandemic"
}

\section{Marek Kucbel ${ }^{1}$}

1 Technical University of Mining and Metallurgy Ostrava

Potential competing interests: The author(s) declared that no potential competing interests exist.

It is a very interesting paper. I recommend the authors to add more explanations about black carbon. Fig. 1 is less easy for readers to read and understand. The gray scale (in Flg. 1) is not satisfactory. 